\title{
Epilepsy surgery in children and adolescents: Report on 43 cases
}

\author{
Marina Aberastury, M.D. ${ }^{a}$, Betina Comas, M.D. ${ }^{a}$, María García, M.D. ${ }^{a}$, Ana Besocke, M.D. ${ }^{a}$, Carlos Ciraolo, M.D. ${ }^{a}$, \\ Guillermo Agosta, M.D. ${ }^{a}$ and Walter Silva, M.D. ${ }^{a, b}$
}

\begin{abstract}
Epilepsy surgery in children with refractory epilepsy is one of the most effective methods to control seizures. The proper selection and assessment of surgery candidates is critical for surgical treatment to be adequately effective and safe. The purpose of this article is to describe our experience with 43 consecutive pediatric patients that underwent epilepsy surgery for refractory epilepsy between September 2005 and May 2014. Effectiveness, safety, and prognostic factors were analyzed. The median age was 12 years old at the time of surgery and 4.5 years old at epilepsy onset, with a latency period of up to 6 years until surgery. Since the surgery, the 43 patients have been in follow-up for a median of 5.4 years ( \pm 2.3 years).

Resective surgery was performed in 32 patients and hemispherectomy, in 11 patients. To date, $62.8 \%$ of patients remain seizure-free. A better prognosis was observed in patients who underwent surgery with a duration of epilepsy of less than two years and in patients in whom a complete resection of the epileptogenic zone was achieved.

Key words: refractory epilepsy, surgery, cortical dysplasia, hemispherectomy, child.
\end{abstract}

http:/ /dx.doi.org/10.5546/aap.2016.eng.458

\section{INTRODUCTION}

The worldwide prevalence of epilepsy is $7 / 1000$ inhabitants, and its incidence ranges between 20 and 70 per 100,000 new cases per year. Epilepsy prevalence has been estimated to be $15-20$ per 1000 inhabitants in developing countries, compared to 4-7 per 1000 inhabitants in developed countries.

a. Hospital Italiano de Buenos Aires.

b. Instituto Argentino de Investigación Neurológica.

E-mail address:

Marina Aberastury, M.D.: marina.aberastury@hiba.org.ar

Funding: None.

Conflict of interest: None.

Received: 10-8-2015

Accepted: 3-9-2016
In the pediatric population younger than 15 years old, epilepsy incidence has been reported to be 89 per 100,000 inhabitants; among them, between $18 \%$ and $54 \%$ have their first seizure before turning 10 years old.

In spite of advances made in the medical management of epilepsy, it has been estimated that $20-30 \%$ of patients have drug resistant epilepsy (DRE). ${ }^{1}$

Over the past years, the safety and effectiveness of epilepsy surgery for the management of children with medically intractable epilepsy have been well established. However, publications including pediatric patients in Argentina are scarce due to the limited number of medical facilities capable of providing this therapeutic option in our setting.

In 2003, the first practice standards on neocortical resections for refractory epilepsy were published. ${ }^{2}$ Their objective was to promote and monitor the increasing number of surgeries and their early indication.

Contrary to the important advances made in developed countries, these have been dissimilar in developing countries. The first limitation arises in connection with barriers to access pre-surgical assessments. $^{3}$

The selection of refractory epilepsy patients who may be candidates for surgical treatment requires an interdisciplinary management, which should include an epileptologist, a neuropsychologist, neurophysiologist, neuroradiologist, and a neurosurgeon.

Patients should have a comprehensive diagnostic assessment to establish the origin and propagation of abnormal electrical activity and thus detect the epileptogenic zone causing seizures. ${ }^{4}$

Some patients with refractory epilepsy require invasive assessment methods to establish the origin of the epileptogenic zone. The most common indications include multiple epileptogenic zones, focal injuries close to or in an eloquent area, and no visible epileptogenic lesion in the brain magnetic resonance imaging (MRI). 
Neurosurgical procedures for treating refractory epilepsy include:

- Curative procedures

- Resective surgeries

- Disconnective techniques:

Hemispherectomy

Multiple subpial transection

- Palliative procedures

- Corpus callosotomy

- Neuromodulation techniques

Surgery success depends, to a great extent, on the possibility to achieve complete epileptogenic tissue resection. ${ }^{5}$

\section{OBJECTIVES}

To describe our experience with pediatric patients that underwent epilepsy surgery for refractory epilepsy.

To analyze the effectiveness, safety and prognostic factors of our epilepsy surgery program for children and adolescents.

\section{MATERIAL AND METHODS Our experience}

We conducted a prospective, cross-sectional, analytical study of 43 consecutive pediatric patients with refractory epilepsy who underwent a surgical procedure, either resective or disconnective, before turning 21 years old at the Department of Pediatric Neurology of Hospital Italiano de Buenos Aires and the Argentine Institute of Neurological Research (Instituto Argentino de Investigación Neurológica), in Buenos Aires (Argentina) between September 2005 and May 2014.

Patients with post-operative follow-up for less than one year or lost to follow-up in the past 12 months were excluded.

\section{Pre-surgical assessment protocol}

Pre-surgical assessment included invasive and non-invasive tests.

The latter included recording the patient's case history, a neurological physical exam, ictal semiology analysis, neuroimaging procedures, and a prolonged surface videoelectroencephalographic (EEG) monitoring. All patients had a neuropsychological assessment, a psychiatric evaluation and 1.5 and 3 tesla highresolution MRIs.

In addition, functional neuroimaging, positron emission tomography (PET), or single photon emission computed tomography (SPECT) were used for patients with an inadequate correlation between the surface video-EEG and the brain MRI or those with no visible epileptogenic lesion in the brain MRI.

Invasive procedures included the placement of deep electrodes and/or subdural grids for invasive monitoring of seizures and cortical functional mapping.

Surgeries were classified as hemispherectomy (disconnective) and resective; the latter were sub-classified as frontal, temporal, or posterior (parieto-occipital).

\section{Prognosis}

Seizure prognosis was assessed using the modified Engel scale (Table 1).

Post-operative neurological deficits and complications were assessed one week, one month and one year after surgery.

\section{Ethical considerations}

Ethical principles for health research were followed to record data and prepare the manuscript. No experimental therapeutic interventions were conducted. Epidemiological results were published protecting patients' identity. Informed consents were obtained.

\section{Statistical analysis}

Continuous outcome measures were expressed as mean and standard deviation (SD) or as median, depending on their distribution; categorical outcome measures were described as proportions.

Prognosis was analyzed based on the modified Engel scale and categorized as excellent (Engel I), or fair or poor (Engel II-III-IV). The univariate analysis was done using the $\chi^{2}$ test, Fisher's exact test, and Wilcoxon rank-sum test to compare results. Age, etiology, duration, epilepsy location, presence of epileptogenic lesion in the MRI, invasive neurophysiological techniques, functional mapping using direct electrical stimulation, resection type, complete lesion resection, and complete epileptogenic zone resection were analyzed. ${ }^{5}$

Age at the time of surgery, epilepsy location, and epilepsy duration before surgery were analyzed as effect modifiers.

Also, 95\% confidence intervals (CIs) were obtained. A value of $p<0.05$ was considered statistically significant.

\section{RESULTS}

Forty-three patients were included; distributed 
by sex, there were 20 girls ( $46.5 \%$ ) and 23 boys (53.5\%). Twenty-seven patients $(64.3 \%)$ had mental retardation before surgery.

Patients' median age at the time of surgery was 12 years old. Their median age at the time of seizure onset was 4.5 years old (range: $0-14$ ), and 11 patients $(25.6 \%)$ had had their first seizure before 1 year old. The median interval between the first seizure and surgery was 6 years, with a minimum interval of 5 months and a maximum of 19 years.

Out of the 43 patients included in the study, 21 $(48.8 \%)$ had focal cortical dysplasia (FCD) as the etiology of epilepsy. In this sub-population, age at seizure onset ranged between 1 week old and 14 years old (median: 3 years old), and epilepsy duration before surgery was 7 years. Their median age at the time of surgery was 14 years old (range: 2-21 years old).

No alterations were observed in the MRIs of four of these patients (19.05\%). Invasive monitoring and resective surgery were performed in 18 patients $(90 \%)$ while disconnective techniques were used in two.

\section{Effectiveness}

Prognosis analysis indicated that 27 patients $(62.8 \%)$ remained seizure-free, with a follow-up of 5.4 years ( \pm 2.3$)$. A worse prognosis was observed among patients with frontal lobe epilepsy.
In our series, only three patients were diagnosed with hippocampal sclerosis; all corresponded to Engel IA class. Fourteen patients in our series underwent surgery with an epilepsy duration shorter than two years; all corresponded to Engel I or II class, $p=0.03$ (Tables 2 and 3).

Among patients who required hemispherectomies, $82 \%$ remained seizure-free.

Surgery was indicated in two patients for the management of status epilepticus, both refractory to multiple antiepileptic agents, including druginduced coma in one patient, and ketogenic diet in the other. One patient underwent a disconnective surgery and remained seizure-free while the other had a resective procedure and was classified as Engel II.

Epileptogenic zone resection was incomplete in six patients $(13.9 \%)$ because it extended into eloquent cortex areas. Only one of the six patients in whom complete resection of the epileptogenic zone as demarcated by invasive neurophysiological techniques could not be achieved remained seizure-free, Engel IA class, with follow-up for 8.2 years, $p=0.01$.

In the FCD group, 11 patients $(52.3 \%)$ remained seizure-free, Engel IA class, with follow-up for 4.7 years $( \pm 2.5), p=0.58$. The analysis of patients with FCD type II indicated that $7 / 13$ (53.8\%) were Engel I class, and 15.4\%, Engel II.

TABLE 1: Modified Engel scale

CLASS I Free of disabling seizures (excluding the postoperative period, one month)

A Completely seizure-free since surgery.

B Only simple partial seizures.

C Some seizures after surgery, but free of seizures for at least two years.

D Generalized seizures with antiepileptic drug withdrawal only.

CLASS II Rare disabling seizures (almost seizure-free)

A Initially free of seizures but has rare seizures now.

B Rare seizures since surgery.

C More than rare seizures after surgery, but rare seizures for at least two years.

D Nocturnal seizures only.

CLASS III Worthwhile improvement

A Worthwhile seizure reduction.

B Prolonged seizure-free intervals amounting to greater than half the follow-up period, but not less than two years.

\section{CLASS IV No worthwhile improvement}

A Significant seizure reduction (between $50 \%$ and $90 \%$ ).

B No appreciable change.

C Worsening of seizures. 
No statistically significant differences were observed in this group when analyzing the relationship between prognosis and epilepsy duration before surgery. Based on the new FCD classification established by the International League Against Epilepsy (ILAE), the following anatomic pathology results were observed: 11 patients $(52.38 \%)$ had FCD type IIa; $4(19 \%)$, FCD type $\mathrm{Ib} ; 2$ (9.5\%), FCD type Ia; 2 (9.5\%), FCD type IIlb; and $2(9.5 \%)$, FCD type IIIb. ${ }^{6}$

\section{Course}

After analyzing complications, it was observed that 19 patients had a new post-operative neurological deficit, which was transient in 8 $(18.6 \%)$ and permanent in $14(32.6 \%)$. Among the latter, the deficit was consistent with the resected area in 11.

Three patients had an unexpected permanent deficit based on the resected area; all of them had motor impairment, and two had FCD in the premotor cortex. Invasive neurophysiological techniques were required in three patients, $p=0.01$.

Four patients required a ventriculoperitoneal shunt due to hydrocephalus; two of them had undergone a hemispherectomy.

Seven patients $(16.3 \%)$ had post-operative infections: three, surgical wound infection (two required bone flap removal); one, subdural empyema; two, bacterial meningitis; and one, intracranial bacterial abscess. Patients who did not require invasive neurophysiological techniques did not develop infections, $p=0.000$.

\section{DISCUSSION}

The purpose of epilepsy surgery is to remove the epileptogenic zone or disconnect the epileptogenic network that is causing seizures without generating new neurological deficits or worsening existing ones.

Post-operative prognosis depends on multiple outcome measures. One is epilepsy duration before surgery. In our series, the median latency period between the first seizure and surgery was six years; three years for hemispherectomies, and seven for resective surgeries. An international multicenter study demonstrated that only one-third of children with refractory epilepsy underwent surgery, even though epilepsy had developed less than two years before in more than $60 \%$ of cases. Consistent with what has been reported, we observed a better prognosis in patients who had the surgery within two years of epilepsy onset. ${ }^{7}$

In our series, it was observed that $69.2 \%$ of temporal epilepsy cases and $47.3 \%$ of extratemporal epilepsy cases corresponded to Engel I class. It is worth noting that, when analyzing results, prognostic factors vary depending on whether surgery was indicated for temporal or extratemporal epilepsy. A meta-analysis that included 36 studies and 1259 pediatric patients with extratemporal epilepsy showed that post-operative prognosis corresponded to Engel I class in $56 \%$ of patients; another meta-analysis that included 36 studies and 1318 pediatric patients with temporal epilepsy showed that post-operative prognosis was Engel I in 76\% of them. ${ }^{8,9}$ Such difference in the percentage of Engel I patients is likely due to the fact that, in our population, there was a high percentage of FCD and a prolonged latency period until surgery. Both factors were associated, in different series, with a worse post-operative seizure prognosis. ${ }^{4,5}$

TABLE 2: Population characteristics based on surgery location

\begin{tabular}{|c|c|c|c|c|c|c|c|c|}
\hline \multirow[b]{2}{*}{ Location } & \multicolumn{2}{|c|}{ Frontal } & \multicolumn{2}{|c|}{ Temporal } & \multicolumn{2}{|c|}{ Posterior } & \multicolumn{2}{|c|}{ Hemispheric } \\
\hline & 15 & $34.9 \%$ & 13 & $30.2 \%$ & 4 & $9.3 \%$ & 11 & 25.6 \\
\hline Age at the time of surgery & 13 & Range: 0-12 & 17 & Range: 0.4-14 & 14 & Range: 6-12 & 8 & Range: $0.1-12$ \\
\hline Age at epilepsy onset & 4 & Range: 2-21 & 2 & Range: 1-21 & 7 & Range: $14-16$ & 4 & Range: 3-21 \\
\hline Latency period, in years & 6 & Range: $0.5-16$ & 12 & Range: 0.5-19 & 7 & Range: 2-10 & 3 & Range: $1-18$ \\
\hline Cortical dysplasias & 12 & $80 \%$ & 6 & $46.2 \%$ & 1 & $25 \%$ & 2 & $18.2 \%$ \\
\hline \multirow[t]{2}{*}{ Engel I } & 6 & $40 \%$ & 9 & $69.2 \%$ & 3 & $75 \%$ & 9 & $81.9 \%$ \\
\hline & \multicolumn{2}{|c|}{$p=0.02$} & \multicolumn{2}{|c|}{$p=0.56$} & \multicolumn{2}{|c|}{$p=0.6$} & \multicolumn{2}{|c|}{$p=0.13$} \\
\hline Normal MRI & 2 & 13.35 & 3 & $23 \%$ & 0 & $0 \%$ & 0 & $0 \%$ \\
\hline Invasive monitoring & 14 & $93.3 \%$ & 12 & $92.3 \%$ & 4 & $100 \%$ & 1 & 9.1 \\
\hline Cortical mapping & 11 & $73.3 \%$ & 8 & $61.5 \%$ & 4 & $100 \%$ & 0 & \\
\hline
\end{tabular}

MRI: magnetic resonance imaging. 
Although only three of our patients had been diagnosed with hippocampal sclerosis, it was observed that all remained seizure-free (Engel IA), similar to what has been reported by a different local series that included 38 pediatric patients, $92 \%$ of whom corresponded to Engel IA class. ${ }^{10}$

As reported in the literature, patients with

TABLE 3: Sample characteristics

\begin{tabular}{|c|c|c|}
\hline Characteristics & $\mathrm{N}=43$ & \\
\hline \multicolumn{3}{|l|}{ Sex } \\
\hline Female & $23(53.5 \%)$ & $95 \%$ CI (0.3765473-0.6882381) \\
\hline Male & $20(46.5 \%)$ & $95 \%$ CI (0.3117619-0.6234527) \\
\hline Age (years old) & Median: 12 & Rango $=1-21$ \\
\hline$\leq 10$ years old & $20(46.5 \%)$ & 95\% CI (0.3117619-0.6234527) \\
\hline$>10$ years old & $23(56.4 \%)$ & 95\% CI (0.3765473-0.6882381) \\
\hline Age at epilepsy onset (years old) & Mean: 4 & Range $=0-14$ \\
\hline Epilepsy onset-surgery interval & Median: 6 & Range $=0.5-19$ \\
\hline \multicolumn{3}{|l|}{ MRI } \\
\hline Evidence of injury & $38(88.4 \%)$ & 95\% CI (0.7491676-0.9611477) \\
\hline No evidence of injury & $5(11.6 \%)$ & 95\% CI (0.0388523-0.2508324) \\
\hline \multicolumn{3}{|l|}{ Epileptogenic zone } \\
\hline Fronto-central & $15(343.9 \%)$ & $95 \%$ CI (0.2100782-0.5092664) \\
\hline Temporal & $13(30.2 \%)$ & $95 \%$ CI (0.171825-0.4612533) \\
\hline Posterior (occipital and parietal) & $4(9.3 \%)$ & $95 \%$ CI (0.0259313-0.2213534) \\
\hline Hemispheric & $11(25.6 \%)$ & $95 \%$ CI (0.135186-0.4117157) \\
\hline \multicolumn{3}{|l|}{ Etiology } \\
\hline Dysplasia & $20(48.8 \%)$ & $95 \%$ CI (0.3117619-0.6234527) \\
\hline Gliosis & $9(20.9 \%)$ & $95 \%$ CI (0.1004411-0.3604248) \\
\hline Tumor & $6(14 \%)$ & $95 \%$ CI (0.0529766 - 0.2793248$)$ \\
\hline Rasmussen syndrome & $4(9.3 \%)$ & $95 \%$ CI (0.0259313-0.2213534) \\
\hline Hippocampal sclerosis & $3(7 \%)$ & 95\% CI (0.0146255-0.1906072) \\
\hline DNT & $1(2.3 \%)$ & 95\% CI (0.0005886-0.1228905) \\
\hline \multicolumn{3}{|l|}{ Neurophysiology } \\
\hline Non-invasive & $12(27.9 \%)$ & $95 \%$ CI (0.1532892-0.436687) \\
\hline Invasive & $31(72.1 \%)$ & $95 \%$ CI (0.563313-0.8467108) \\
\hline Grids + deep electrodes & $4 / 31(9.3 \%)$ & 95\% CI (0.0363017-0.2983358) \\
\hline Grids & $14 / 31(32.6 .1 \%)$ & $95 \%$ CI (0.273165-0.6396577) \\
\hline Deep electrodes & $13 / 31(30.23 \%)$ & $95 \%$ CI (0.245476-0.6092408) \\
\hline \multicolumn{3}{|l|}{ Surgery typ } \\
\hline Corticectomy & $11(25.6 \%)$ & $95 \%$ CI (0.135186-0.4117157) \\
\hline Corticectomy and lesionectomy & $7(16.3 \%)$ & 95\% CI (0.0680521-0.3070109) \\
\hline Lesionectomy & $2(4.6 \%)$ & $95 \%$ CI (0.0056833-0.1581115) \\
\hline Lobectomy & $4(9.3 \%)$ & $95 \%$ CI (0.0259313-0.2213534) \\
\hline Standard ATL & $7(16.3 \%)$ & $95 \%$ CI (0.0680521-0.3070109) \\
\hline Tonsillectomy & $1(2.3 \%)$ & 95\% CI (0.0005886-0.1228905) \\
\hline Hemispherectomy & $11(25.6 \%)$ & $95 \%$ CI (0.135186-0.4117157) \\
\hline Complications & $8(18.6 \%)$ & 95\% CI (0.0839124-0.3340145) \\
\hline Transient deficit & $8(18.6 \%)$ & 95\% CI (0.0839124-0.3340145) \\
\hline Permanent deficit & $14(32.6 \%)$ & 95\% CI (0.1907628-0.4854398) \\
\hline Hydrocephalus & $4(9.3 \%)$ & 95\% CI (0.0259313-0.2213534) \\
\hline Infection & $6(14 \%)$ & $95 \%$ CI $(0.0529766-0.2793248)$ \\
\hline Death & $0(0.00 \%)$ & $95 \%$ CI $(0-0.0822111)^{*}$ \\
\hline \multicolumn{3}{|l|}{ Engel } \\
\hline I & $27(62.8 \%)$ & 95\% CI (0.4672509-0.7702483) \\
\hline II & $8(18.6 \%)$ & 95\% CI (0.0839124-0.3340145) \\
\hline III (acceptable) and IV (worse) & $8(18.6 \%)$ & 95\% CI (0.0839124-0.3340145) \\
\hline
\end{tabular}

MRI: magnetic resonance imaging; DNT: dysembryoplastic neuroepithelial tumor; CI: confidence interval; ATL: anterior temporal lobectomy. 
focal epilepsy outside the frontal lobe in whom a complete resection of the epileptogenic zone was achieved had a better prognosis. ${ }^{4}$

In our series, the percentage of remission in patients who required an hemispherectomy was marginally greater than that reported in other international and national series. ${ }^{11,12}$

In this series, as in other literature publications, transient and permanent complications were observed; among the latter, $7 \%$ were unexpected and secondary to surgery complications.

Malformations of cortical development account for a range of structural and functional abnormalities that may cause epilepsy. For example, FCDs, dysembryoplastic neuroepithelial tumors, and gangliogliomas, which may be subjected to surgical treatment.

It is worth noting that FCDs are the main etiology in refractory epilepsy cases among pediatric surgery candidates..$^{13}$ In our population, FCD was the etiology in $48.8 \%$ of patients. In this group of patients, no FCD was observed in the MRI of $19 \%$ of patients, similar to what has been reported by other authors (14\%-23\%). ${ }^{7,14}$

An excellent post-operative prognosis, Engel I, was established in $52.3 \%$ of our patients.

Reports on the prognosis of epilepsy surgery for FCD have been varied. Some recent series have observed a $32-89 \%$ seizure-free prognosis. ${ }^{13}$

The analysis of a sub-group of patients with FCD type II, usually associated with a better prognosis, indicated that $53.8 \%$ were Engel I class, and $15.4 \%$, Engel II. Similar results were observed in other international series while an Argentine series demonstrated better results, with $67.7 \%$ of patients classified as Engel I. ${ }^{13,15}$

\section{Limitations}

The small number of patients included in this study prevented an adequate multivariate analysis and certain subgroup analyses.

This study did not analyze seizure semiology or electroencephalogram findings.

\section{CONCLUSION}

In our program, epilepsy surgery was associated with a favorable prognosis: almost two-thirds of patients remained seizure-free.

A better prognosis was observed in patients who underwent surgery with a duration of epilepsy of less than two years and in patients in whom a complete resection of the epileptogenic zone was achieved.

In terms of surgical treatment safety, in our series no mortality was observed and, in relation to morbidity, only a small number of patients had long-term complications.

\section{REFERENCES}

1. Kwan P, Arzimanoglou A, Berg AT, Brodie MJ, et.al. Definition of drug resistant epilepsy: consensus proposal by the ad hoc Task Force of the ILAE Commission on Therapeutic Strategies. Epilepsia 2010;51(6):1069-77.

2. Engel JJ, Wiebe S, French J, Sperling M, et al. Practice parameter: temporal lobe and localized neocortical resections for epilepsy: report of the Quality Standards Subcommittee of the American Academy of Neurology, in association with the American Epilepsy Society and the American Association of Neurological Surgeons. Neurology 2003;60(4):538-47.

3. Qiu J.Epilepsy surgery: challenges for developing countries. Lancet Neurol 2009;8(5):420-1.

4. Ryvlin P, Cross JH, Rheims S. Epilepsy surgery in children and adults. Lancet Neurol 2014;13(11):1114-26.

5. Teutonico F, Mai R, Veggiotti P, Francione S, et al. Epilepsy surgery in children: evaluation of seizure outcome and predictive elements. Epilepsia. Epilepsia 2013;54(Suppl 7):70-6.

6. Blümcke I, Thom M, Aronica E, Armstrong DD, et al. The clinicopathologic spectrum of focal cortical dysplasias: a consensus classification proposed by an ad hoc Task Force of the ILAE Diagnostic Methods Commission. Epilepsia 2011;52(1):158-74.

7. Harvey AS, Cross JH, Shinnar S, Mathern GW. Defining the spectrum of international practice in pediatric epilepsy surgery patients. Epilepsia 2008;49(1):146-55.

8. Englot DJ, Breshears JD, Sun PP, Chang EF, Auguste KI. Seizure outcomes after resective surgery for extra-temporal lobe epilepsy in pediatric patients. I Neurosurg Pediatr 2013;12(2):126-33.

9. Englot DJ, Rolston JD, Wang DD, Sun PP, et al. Seizure outcomes after temporal lobectomy in pediatric patients. J Neurosurg Pediatr 2013;12(2):134-41.

10. Cersósimo R, Flesler S, Bartuluchi M, Soprano AM, et al. Mesial temporal lobe epilepsy with hippocampal sclerosis: study of 42 children. Seizure 2011;20(2):131-7.

11. Caraballo R, Bartuluchi M, Cersósimo R, Soraru A, Pomata H. Hemispherectomy in pediatric patients with epilepsy: a study of 45 cases with special emphasis. Childs Nerv Syst 2011;27(12):2131-6.

12. Lee YJ,Kim EH, Yum MS, LeeJK, etal. Long-Term Outcomes of Hemispheric Disconnection in Pediatric Patients with Intractable Epilepsy. J Clin Neurol 2014;10(2):101-7.

13. Lüders H, Schuele SU. Epilepsy surgery in patients with malformations of cortical development. Curr Opin Neurol 2006;19(2):169-74.

14. Fauser S, Essang C, Altenmüller DM, Staack AM, et al. Long-term seizure outcome in 211 patients with focal cortical dysplasia. Epilepsia 2015;56(1):66-76.

15. Noli D, Bartuluchi M, González FS, Kaltenmeier MC, et al. Type II focal cortical dysplasia: electroclinical study and surgical outcome in 31 pediatric patients. Childs Nerv Syst 2013;29(11):2079-87. 